\title{
PLATAFORMAS DIGITALES Y CONCEPTO DE TRABAJADOR: UNA PROPUESTA DE INTERPRETACIÓN FINALISTA
}

\author{
Digital Platforms and the concept of worker: \\ Personal work relationship as a concept for workers \\ Adrian Todolí Signes* \\ Universidad de Valencia
}

\section{RESUMEN}

Existe consenso en la doctrina laboralista de que el concepto de trabajador debe interpretarse conforme a la realidad social del momento en el qué debe ser aplicado. El presente trabajo analiza qué significa esta afirmación en el momento actual de expansión de las plataformas digitales y del trabajo autónomo en general. Para ello, se analizan i) las propuestas existentes en la doctrina comparada de ampliación del ámbito subjetivo del Derecho del trabajo; ii) los fines propios del Derecho del trabajo; iii) las últimas interpretaciones del Tribunal Supremo del concepto de trabajador. En base a este análisis, se realiza una propuesta de interpretación finalista del concepto de trabajador que permita incluir a todos aquellos que prestan servicios sin ser genuinamente una empresa.

Palabras clave: Concepto de trabajador, ámbito subjetivo de aplicación del estatuto de los trabajadores, subordinación, dependencia, ajenidad, interpretación finalista del concepto de trabajador.

\section{ABSTRACT}

There is consensus in the labor law doctrine that the concept of worker must be interpreted according to the social reality of the moment in which it should be applied. The present work analyzes what this affirmation means in the current moment of expansion of digital platforms and autonomous work in general. For that, i) the existing proposals in the comparative doctrine of extension of the subjective scope of Labor Law are analyzed; ii) the aims of labor law; iii) the latest interpretations of the Supreme Court of the concept of worker. Based on this analysis, a purposive interpretation proposal is made of the concept of worker that allows to include all those who provide services without being genuinely a company.

Keywords: worker concept, Labour Law scope, subordination, control-test, finalist interpretation of the concept of worker.

* Correspondencia a: Adrián Todolí Signes. Facultad de Derecho. Departamento de Derecho del Trabajo. Av. Tarongers s(n 46020VALENCIA, España - adrian.todoli@uv.es - https://orcid.org/0000-0001-7538-4764

Cómo citar: Todolí Signes, Adrián. (2019). «Plataformas digitales y concepto de trabajador: una propuesta de interpretación finalistaw; Lan Harremanak, 41, 17-41. (https://doi.org/10.1387/lan-harremanak.20880).

Recibido: 22 mayo, 2019; aceptado: 25 junio, 2019.

ISSN 1575-7048 - elSSN 2444-5819 / (C) 2019 UPV/EHU 


\section{SUMARIO}

1. Las plataformas y los retos del Derecho del trabajo.-2. El creciente trabajo en plataformas: tipos de trabajos y sectores.-3. La digitalización y la desigualdad. - 4. Más allá de los trabajadores de plataformas: la ampliación del ámbito subjetivo de protección del Derecho del trabajo.-5. Una interpretación finalista del concepto de trabajador laboral.-6. La labor del Tribunal Supremo en la reinterpretación del concepto de trabajador.-7. Reflexión final.-8. Bibliografía.

\section{Las plataformas y los retos del Derecho del trabajo}

Una de las mayores innovaciones del trabajo en plataformas ha sido su capacidad de producir una nueva forma de organización del trabajo. La tecnología actual (aplicaciones, páginas web, smartphones, algoritmos, entre otros) permite nuevos modelos de negocio y de prestación de servicios que despiertan el interés de los consumidores ${ }^{1}$.

No es la primera vez que se produce una situación similar vinculada, precisamente, a los cambios tecnológicos. Con la máquina de vapor y los telares mecanizados, las formas de producción y distribución de textiles cambiaron radicalmente. Antes de la industrialización existía una producción individualizada en los hogares de los trabajadores, de donde el empresario recogía semanalmente (o con otra periodicidad) las prendas para, a continuación, distribuirlas en el mercado. Posteriormente, con las posibilidades que ofrecía la tecnología, se cambió a una producción en fábricas. En efecto, el incremento de productividad provocado por el telar mecánico hacía económicamente ineficiente continuar cosiendo individualmente vestidos por los trabajadores en sus respectivas casas. Por el contrario, era necesario realizar fuertes inversiones en la adquisición de maquinaria y aquellos que no podían costearla — quienes vivían de su trabajo- debían abandonar el trabajo doméstico para acudir a la fábrica y ofrecer su tiempo a cambio de una remuneración. En base a esta forma de prestar servicios, y con objeto de incrementar la eficiencia del proceso productivo, apareció

1 Un reciente informe de la Comisión Europea establece tres tipos de organización la economía industrial de 1850 a 1970, la economía de la información de 1970 a 2020 y un tercer tipo llamado economía de la inteligencia artificial que empezando en los 2010 será imperante en el futuro. Esta economía de la inteligencia artificial incluye en su seno la economía de plataformas, pero también el big data, ver Goos (2019). 
— con el tiempo — la cadena de montaje fordista y, persiguiendo la reducción de los costes de mano de obra, el taylorismo.

Precisamente, debido a este cambio en la forma de organizar el trabajo - del trabajo en casa a la fábrica - provocado por la necesidad del uso de la tecnología, de la cual los trabajadores no eran titulares, aparecieron los primeros conflictos sociales enfrentando a los empresarios — propietarios de la tecnología indispensable - con los trabajadores — que no la poseían-. Esta posición de privilegio del empresario permitió abusos sobre los trabajadores, quienes habían de someterse a las órdenes del titular de los medios de producción, a jornadas extenuantes marcadas por éste y a precarias condiciones de salubridad en las fábricas si querían continuar trabajando. Frente a ello, surgió el Derecho del trabajo como forma de «resolver» el conflicto entre las partes con, entre otros, los siguientes objetivos (Davidov, 2002).

— La protección de la parte débil frente abusos (desigualdad del poder de negociación)

— La dignificación del trabajo y sus condiciones (valores sociales)

- La pacificación y encauzamiento del conflicto colectivo dentro del sistema capitalista (fallos de mercado)

Así pues, el nacimiento del Derecho del trabajo es indisociable de la industrialización y de la aparición de grandes fábricas con trabajadores que realizan sus funciones bajo la estricta supervisión de los encargados. Dado el interés del legislador original de proteger a este sujeto que prestaba servicios en el marco de una organización determinada, se diseñó el concepto legal de "trabajador» para describirlo. A su vez, el sistema de protecciones (jornada máxima, salario mínimo, etc.) se creó pensando en las necesidades de estos trabajadores y los derechos laborales se limitaron a aquéllos que eran compatibles con el sistema de producción industrial, otorgando ciertas potestades de control de los trabajadores al empresario (entre otros, su horario, su jornada, instrucciones directas, etc.). En conclusión, tanto el concepto «trabajador», como su régimen jurídico, fueron pensados y adaptados a ese modelo de organización empresarial/industrial.

En este contexto, y con la legislación de inspiración industrial todavía vigente, aparece una nueva forma de organizar el trabajo, que no surge aisladamente, sino que culmina un proceso de descentralización productiva y «desverticalización» de las empresas, que proviene de los ańos 70 . Efectivamente, la empresa integrada verticalmente ha sido remplazada por una empresa-red mucho más dinámica y adaptable a las necesidades del mercado en cada momento. Las empresas que aportan un alto valor ańadido ya no desean integrar todos los elementos del proceso productivo en su matriz, sino mantener exclusivamente el núcleo del negocio para descentralizar y subcontratar en otras empresas el resto del proceso. Ello les permite adaptar su estructura de costes a la necesidad del mercado con mucha facilidad. Así, rescindir una contrata con otra empresa cuando una parte del proceso 
productivo ya no resulta necesario es mucho menos costoso que escindir o extinguir parte de la empresa propia (Calvo, 2017). De esta forma, este proceso de descentralización ha creado empresas de "primera" — las que mantienen el valor productivo - y de «segunda» — servicios accesorios—, modelo al cual el Derecho del trabajo ha ido adaptándose, sin muchas modificaciones, como ha podido. Esto ha sido posible dado que la descentralización productiva aquí narrada se limitaba a cambiar la forma de organizar las empresas, pasando de una integración vertical a una coordinación entre ellas o incluso a una subordinación de unas empresas a otras, pero sin afectar al núcleo de la prestación del trabajo.

En este proceso de descentralización aparece el trabajo en plataformas como expresión última de dicha descentralización productiva. No obstante, a diferencia de las anteriores manifestaciones del fenómeno, esta vez no se afecta solamente a la forma en que las empresas se organizan y se coordinan, sino que también repercute directamente en cómo se organiza el trabajo, esto es, cómo se presta el servicio por parte del trabajador y cómo se relaciona la empresa cliente final con la persona que presta el servicio.

En este punto se plantea la cuestión de si el concepto de trabajador, o más bien el propio ámbito subjetivo del Estatuto de los Trabajadores, se adecúa a esta nueva realidad social. Tradicionalmente se ha mantenido que el concepto de trabajador es suficientemente flexible como para adaptarse a las nuevas profesiones y a las nuevas realidades económicas sin necesidad de cambios. En efecto, el concepto de trabajo subordinado lleva años acomodándose a las diferentes situaciones, sin que el hecho de que el trabajador tenga libertad para elegir cuándo realiza el trabajo - o cómo- sea relevante para su calificación jurídica. Es precisamente la elasticidad del concepto la que permitirá su adecuación a la «realidad social del tiempo» en el que ha de ser interpretado (Pérez, 1993: 39). Así pues, la pregunta que se pretende responder en este trabajo es ¿qué significa esa elasticidad aplicada actualmente? ¿Cómo se debe interpretar el concepto de trabajador - y a la vez el ámbito de aplicación del Estatuto de los Trabajadorespara responder a las nuevas realidades? Para responder a esta cuestión, el trabajo se ha dividido de la siguiente manera: el apartado dos tratará de configurar una radiografía del trabajo en plataformas en nuestro país con objeto de conocer su intensidad y su alcance; el tercero tratará brevemente de justificar la necesidad de proteger a los trabajadores en plataformas debido a que pertenecen todos a un grupo de proveedores de servicios que requieren de una tecnología - la plataforma-, que no es de su propiedad, ni está bajo su control, para poder prestar sus servicios y los efectos que ello tiene. El cuarto apartado, parte de la premisa de que los trabajadores en plataformas no son los únicos afectados por la desregulación existente en materia de condiciones de trabajo para los trabajadores autónomos, por lo que se plantearán posibles soluciones más globales, entre ellas, un nuevo ámbito de aplicación de Derecho del trabajo que comprenda toda prestación personal de servicios. El quinto, analiza una interpretación finalista 
del concepto de trabajador dependiente que incluya a todos aquellos prestadores personales de servicios — sean dependientes o no- que no sean genuinamente una empresa. El sexto capítulo analizará la última jurisprudencia del Tribunal Supremo en materia de concepto de trabajador para establecer una comparativa con esta doctrina (interpretación finalista del concepto de trabajador) vista en el anterior capítulo. El trabajo termina con unas conclusiones.

\section{El creciente trabajo en plataformas: tipos de trabajos y sectores}

Varios estudios se han llevado a cabo en los últimos años con objeto de cuantificar el empleo en plataformas. Uno de los más amplios es el realizado por la encuesta COLLEM, dirigida por Pesole (2018) y elaborada por la Comisión Europea, que ofrece datos de 14 Estados Miembro, incluyendo España, sobre el número de trabajadores de plataformas. Las estimaciones para nuestro país indican que, en promedio, un 11,6\% de la población adulta española ha recurrido alguna vez a las modalidades online de trabajo en plataformas para obtener ingresos, prestando algún tipo de servicio. Sin embargo, menos del 10\% realiza este tipo de actividad frecuentemente. Respecto a cuántos trabajadores dedican un tiempo considerable de horas (al menos 10 por semana) y obtienen una cuantía significativa de ingresos (al menos un 25\% del total) a través del trabajo en plataformas, la cifra se fija en torno al $6 \%$. Por su parte, en cuanto a los trabajadores cuya actividad económica principal es el servicio prestado en la plataforma, esto es, que, al menos, el $50 \%$ de sus ingresos proviene del trabajo en plataformas o dedican a este tipo de trabajo más de 20 horas por semana, la cifra se reduce a aproximadamente un $2 \%$ de la población adulta. Esto es, un 3,7\% de la población ocupada total en España.

Las cifras absolutas revelan que el número de trabajadores en España cuya fuente principal de ingresos son sus servicios en plataformas asciende a 694.000 personas. Se trata de un número nada desdeñable de afectados por esta nueva forma de trabajar que, además, se espera que aumente exponencialmente en un periodo relativamente corto, considerando que, hace cinco años, esta forma de prestar servicios era prácticamente inexistente en nuestro país.

A mayor abundamiento, la percepción ofrecida por los medios de comunicación podría conducir a la conclusión de que nos encontramos ante un tipo de prestación de servicios que afecta únicamente a un trabajo poco cualificado (transporte, limpieza, etc...), pero nada más lejos de la realidad.

Las plataformas han modificado la forma de organizar el trabajo, y de proveer servicios transversalmente, en todo tipo de sectores y ocupaciones, entre ellos, aquéllos más cualificados. De acuerdo con la encuesta citada, la mayoría de tareas realizadas consisten en funciones administrativas, seguidas inmediatamente por profesiones liberales, como puedan ser abogados, arquitectos, ingenierías o inclu- 
sos servicios médicos. En efecto, el trabajo en plataformas no se limita a ocupaciones poco cualificadas, sino que cualquier prestación de servicios individual es apta para organizarse a través de plataformas. Incluso aquellas que requieren un grupo de trabajadores para completarse pueden organizarse a través de los susodichos sistemas de plataformas, por ejemplo, segmentando el trabajo mediante algoritmos hasta individualizarlo ${ }^{2}$. Así pues, en el sector servicios, pocas ocupaciones escapan a la posibilidad de ofrecerse individualmente a través de plataformas.

A su vez, estos datos nos dan una idea bastante clara de que las plataformas no crean nuevos sectores productivos ni nuevas ocupaciones, sino que esencialmente se limitan a cambiar la forma de ofrecer servicios en el mercado y de organizar el trabajo de profesiones pre-existentes. Y el anterior es hallazgo relevante, puesto que revela que con el trabajo en plataformas no se cumple la promesa schumpeteriana de generación de nuevos sectores productivos gracias a la tecnología creando, así, empleo. En cambio, se trata de una trasformación, en la mayoría de casos, de ocupaciones y empleos ya existentes.

Tipos de trabajos y sectores.

\section{Gráfico 1}

\section{Ocupaciones de los trabajadores en Plataformas}

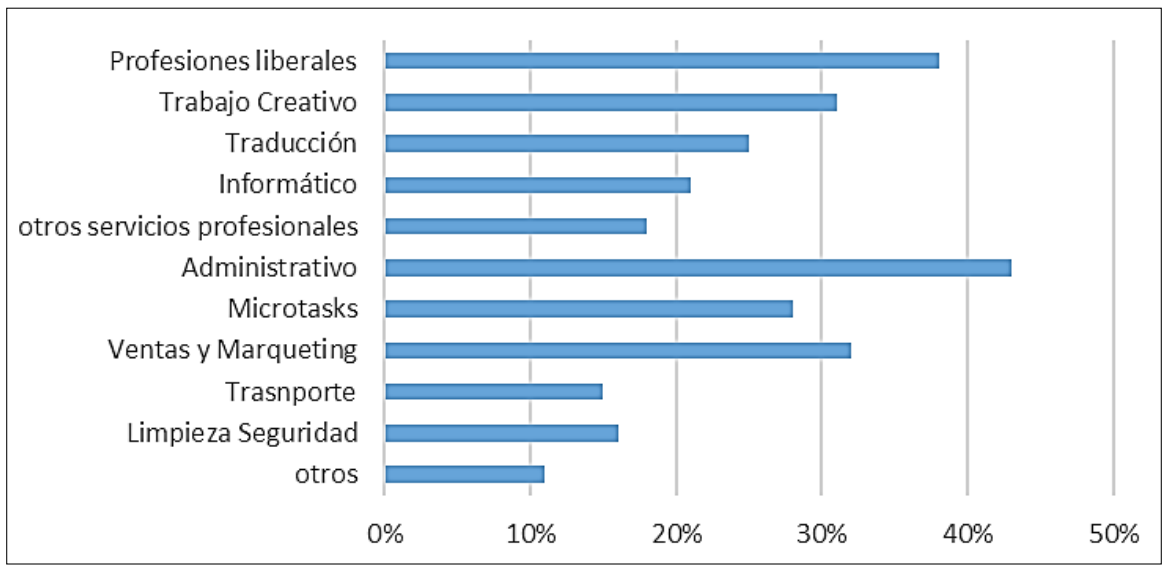

Fuente: elaboración propia a partir de Pesole et al. (2018: 37.38) con datos de la encuesta COLLEEM

2 Por esta razón, desde la doctrina se ha considerado que nos encontramos ante un nuevo taylorismo, Marvit, M., "How Crowdworkers became the ghosts in the digital machine» (en línea), 2014, https:/www.thenation.com/article/how-crowdworkers-became-ghosts-digital-machine/ (consultado 10 de abril de 2019); Todoli Signes, A., El trabajo en la era de la economía colaborativa, Tirant, 2017. 


\section{La digitalización y la desigualdad}

Recientemente, un informe de la Fundación BBVA (2018) alertaba de una fuerte bajada en la participación de las rentas del trabajo en la economía nacional frente a un incremento de las rentas del capital. Lo anterior se traduce en una caída de la masa salarial percibida por los trabajadores españoles. Uno de los principales factores que explica esta reducción es la digitalización. Se argumenta, desde esta teoría, que las nuevas empresas digitales requieren una menor mano de obra en comparación con las empresas tradicionales, generando, incluso, mayores beneficios con un menor número de trabajadores. En efecto, algunos autores han puesto de relieve que la suma de los ingresos obtenidos por GM, Ford y Chrysler en los años 90 es prácticamente idéntica a la declarada por Google, Apple y Facebook, requiriendo estos tres últimos nueve veces menos trabajadores que los anteriores (Tegmark, 2017: 121). Así pues, pocas dudas caben de la importancia de la tecnología en la sustitución del trabajo y como forma de acaparar la mayor parte de los ingresos creados.

Gráfico 2

Participación ajustada de las rentas del trabajo en Espańa 1978-2018

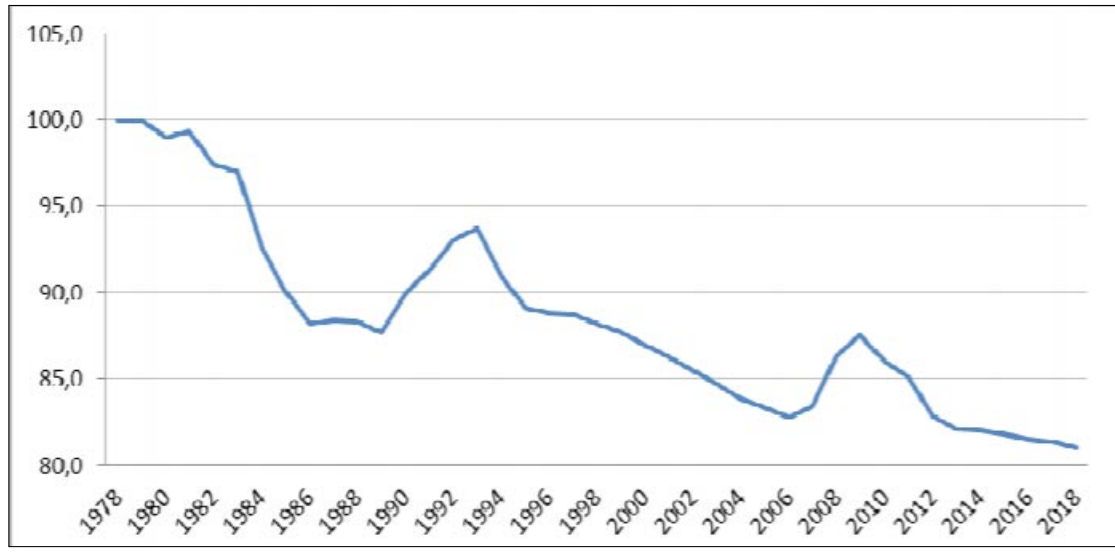

Fuente: Comisión Europea. Base de datos AMECO.

Ello parece confirmarse no solo por la sustitución de los trabajadores por la tecnología, sino también cuando la tecnología se vuelve un instrumento central de la organización del trabajo, como es en el caso de las plataformas digitales. Así, un informe del Instituto JPMorgan Chase (2018), tras analizar los salarios de los conductores de Uber y de otras plataformas digitales del sector del trasporte en 2018 y lo que estos mismos trabajadores percibían en otros empleos en 2013, concluyó que ingresaban significativamente menos en Uber que en sus 
trabajos previos. Concretamente, de media obtenían al mes un 53\% menos que en sus antiguos empleos fuera de la economía de plataformas. Parece, pues, y a falta de más estudios, que el trabajo en plataformas repercute negativamente en los ingresos totales de los trabajadores por su empleo.

Cabe destacar, pues, que la inclusión de la tecnología, y la necesidad de ésta para acceder al mercado disminuye el poder de negociación de aquellos que viven de su trabajo sin ser titulares de la misma -igual que sucedió con los primeros telares-. Esta reducción del poder de negociación incrementa la subordinación del trabajador - que ha de estar a las órdenes de la empresa o perderá la oportunidad de trabajar - a la vez que estrecha la porción de los ingresos totales que se dedican a retribuir el trabajo. Ante esta perspectiva, cabe señalar dos cuestiones.

La primera, y a efectos de lo que aquí interesa, se debe poner en valor la negociación colectiva como forma tradicional de obtener un reparto más equitativo entre las rentas que se destinan al capital (beneficios) y al trabajo (salario) en una empresa o un sector. Cualquier reducción o limitación al uso de este instrumento solamente agravará la inequidad en el reparto de los ingresos obtenidos por las empresas entre los propietarios de la tecnología y los trabajadores que carecen de ella (amén de incrementar la conflictividad laboral ${ }^{3}$ ).

En segundo lugar, visto que los sectores afectados por el trabajo en plataformas son todos aquellos basados en la prestación de un servicio, independientemente de que éste requiera una alta o baja cualificación, y que la economía de plataformas no crea en sí misma un nuevo sector productivo, sino que modifica o transforma los preexistentes y que lo hace incrementando el poder del titular de la plataforma frente al trabajador que carece de ella, cabe la posibilidad de que profesiones liberales, que tradicionalmente funcionaban de manera autónoma y con capacidad de auto-protegerse sin necesidad de intervención del Derecho del trabajo pueden verse ahora sometidos a los mismos riesgos y a la misma —o parecida - subordinación respecto del propietario de la tecnología — y la marca ${ }^{4}$ — que los obreros de las fábricas.

Si las predicciones se cumplen, y los consumidores incrementan la demanda de servicios —incluidas profesiones liberales - a través de las plataformas digitales, estos profesionales liberales dejarán de poder prestar servicios en sus despachos particulares — como tradicionalmente venían haciendo- sin estar integrados en una plataforma. Estas plataformas — que además tienden al monopolio

3 Un claro ejemplo es la huelga a nivel mundial de Uber el 8 de mayo de 2019 ver https:// edition.cnn.com/2019/05/09/tech/uber-driver-deal/index.html (accedido el 13 de mayo de 2019)

${ }^{4}$ La plataforma además de un medio tecnológico consistente en portales web, aplicaciones móviles, algoritmos y recolección de datos para «alimentar los algoritmos» también es una «marca» en el sentido de un elemento distintivo conocido y reconocido por los consumidores necesario para poder acceder al mercado y proveer servicios. 
u oligopolio 5 - ejercerán gran poder de negociación sobre los trabajadores que presten servicios con el uso de su tecnología y marca -igual como lo hicieron los dueños de las fábricas sobre los trabajadores que necesitaban de su maquinaria para poder producir bienes- . En fin, ello puede hacer que estos profesionales, que tradicionalmente prestaban sus servicios de manera independiente, pasen a depender, de una forma u otra, del propietario de la plataforma.

\section{Más allá de los trabajadores de plataformas: la ampliación del ámbito subjetivo de protección del Derecho del trabajo}

El problema de la exclusión de los trabajadores del ámbito de protección del Derecho del trabajo y de la Seguridad Social, sin duda, no es exclusivo de este nuevo modelo de negocio. Es cierto que la aplicación de la tecnología puede, incrementalmente, agravar la situación, además de poseer particularidades dignas de estudio. No obstante, sería ingenuo pensar que se está ante un problema completamente nuevo. Con la evolución del mercado de trabajo de los últimos años se ha constatado un aumento de la sustitución de mano de obra laboral por mano de obra «autónoma». De hecho, la extrema flexibilidad en la utilización de la mano de obra y la transferencia del riesgo desde la empresa hacia el prestador de servicios mediante la utilización de la figura de los autónomos se ha convertido en una realidad para una gran parte de la fuerza de trabajo que va mucho más allá de la economía de plataformas (De Stefano, 2016). Por ello, sería lógico pensar que la prestación de servicios a través de plataformas digitales es sólo parte de una tendencia general hacia la utilización del trabajo autónomo.

Por esta razón, una primera respuesta a la cuestión planteada en este trabajo pasa por ampliar el ámbito subjetivo del Derecho del Trabajo. Es decir, dado que el trabajo en plataformas no es una cuestión aislada, sino que se enmarca en una tendencia generalizada, se podría argumentar que la solución difícilmente puede provenir del estudio particularizado de esta cuestión. Por el contrario, habría que conectar la solución a la falta de protección de los trabajadores en plataformas digitales con aquéllas planteadas para reducir los riesgos que asumen, en general, los trabajadores autónomos que actualmente tampoco quedan cubiertos por la protección del Derecho del trabajo. En definitiva, dado que el problema analizado no es radicalmente nuevo, el análisis de las soluciones doctrinales que se han planteado hasta ahora puede arrojar cierta luz.

En este sentido, las propuestas doctrinales de modernización del Derecho del trabajo elaboradas en los últimos años pasan todas, también, por ampliar del ám-

5 Ver al respecto conclusiones del informe realizado por el IVIE, Pérez García, (2019), 
bito subjetivo de la disciplina. Ello no significa, en todos los casos, ampliar la definición de contrato de trabajo, sino, por el contrario, librar a la especialidad del anclaje del contrato de trabajo como ámbito de aplicación. En efecto, de una forma u otra, las propuestas apuestan porque el Derecho del trabajo no esté constreñido por la definición del contrato de trabajo, sino que su ámbito debería extenderse a todo el «mundo del trabajo» (Freedland y Kountouris, 201121 ). La anterior tendría, como primera consecuencia, que el trabajo personal, esté formulado bajo dependencia o independencia, seguiría sujeto a normativas protectoras.

Las posiciones doctrinales, obviamente, difieren en cómo realizar la ampliación y en el tipo de protecciones que debieran tener las relaciones personales de trabajo que entraran dentro del Derecho del trabajo, pero no dentro del contrato de trabajo.

La Comisión Europea, en un informe realizado bajo la dirección de Alain Supiot (2001), tuvo como objetivo reformular el Derecho del trabajo de tal forma que se liberara de las restricciones del trabajo subordinado bajo el contrato de trabajo. La idea central en el «informe Supiot» fue la de reconocer y concienciar sobre la existencia de un amplio rango de situaciones en las que los ciudadanos están trabajando a pesar de no encontrarse bajo un contrato de trabajo. De hecho, el informe Supiot no se limitaba a ampliar el ámbito subjetivo de la disciplina al "trabajo no subordinado", sino también al «trabajo no remunerado» (ej., cuidados dentro de la familia o tareas domésticas sin retribución). La ambiciosa propuesta configuraba un «statut professionnel» o estatuto profesional, donde se incluirían todos aquellos que prestaran servicios de mano de obra. No obstante, en dicha propuesta, este estatuto se limitaría a conceder derechos de Seguridad Social. Esto es, Supiot proponía desligar la protección de Seguridad Social de la existencia de un contrato de trabajo. Efectivamente, uno de los primeros problemas con los que se encuentran los trabajadores autónomos es su desprotección en materia de Seguridad Social respecto a sus homólogos trabajadores. Por su parte, éste es el campo donde más se avanzado en los últimos años - a pesar de seguir existiendo importantes diferencias-.

También Lyon-Caen (1990: 7-9), en su momento, propuso integrar en el Derecho del Trabajo relaciones personales de trabajo distintas al contrato de trabajo. En este sentido, propuso «un Derecho del trabajo para trabajadores distintos de los existentes en el modelo del Derecho del trabajo». A su vez, enunció qué principios legales debían ser aplicables a trabajadores distintos de los trabajadores subordinados.

Por su parte, Ewing, Hendy y Jones (2016: 35), en su Manifesto for Labour Law y su continuación en la reciente publicación Rolling Out the Manifesto for Labour Law (2018: 36), también sugieren la necesidad de ampliar la construcción nacional del ámbito de aplicación personal de los derechos laborales mediante su propuesta de definición consistente en toda persona contratada por 
otra para proveer trabajo, y que no esté genuinamente dirigiendo una empresa de su propiedad, ha de considerarse trabajador ${ }^{6}$.

Adicionalmente, también Dockés (2017) y otros autores franceses han defendido una ampliación del ámbito de aplicación del Derecho del trabajo en su país.

En una línea similar, es interesante la propuesta realizada por Freedland y Kountouris (2011). Estos autores también se decantan por el abandono de la noción de subordinación como definidor del ámbito del Derecho del Trabajo. Se debe notar que estos autores no proponen la modificación del ámbito subjetivo del contrato de trabajo — que seguiría limitándose a la subordinación,sino que su propuesta es incluir dentro del ámbito del Derecho del trabajo otras relaciones de trabajo personales, que actualmente se encuentran en el derecho privado (Freedland y Kountouris, 2011: 38).

Se parte de la idea de que actualmente se está ante un sistema binario en las prestaciones personales de trabajo; por una parte, el contrato de trabajo — regulado por el Derecho del trabajo— y, por otra, el resto de prestaciones de trabajo amparadas en el Derecho privado. Sin embargo, esta división parte de una falsa idea de que todas las prestaciones de trabajo reguladas por el Derecho civil son idénticas. Este argumento viene reforzado por el hecho de que el Derecho civil, en su regulación, no distingue entre la prestación de servicios personales y los no personales Freedland y Kountouris, 2011: 289). En efecto, la regulación aplicable a un contrato de prestación de servicios entre una empresa y un trabajador autónomo será la misma que entre una empresa y otra empresa. Ante ello, estos autores proponen que el Derecho del trabajo amplíe su ámbito a todas las prestaciones de trabajo realizadas personalmente. Con ello, se pretende que el Derecho del trabajo deje de centrarse en el contrato de trabajo para pasar a centrarse en "la persona que trabaja» (Freedland y Kountouris, 2011:316) ${ }^{7}$.

Retomando esta posición doctrinal se pronuncian Countouris y De Stefano (2019) al establecer que el Derecho del trabajo no debería verse constreñido al trabajo subordinado, puesto que todos aquéllos que "viven de su trabajo» requieren protección. Si bien estos autores en su informe no establecen el nivel de

6 Traducción propia.

7 Por su parte, esta propuesta doctrinal no propugna una equiparación en los derechos y deberes de todas «las personas que trabajan» con los derechos y deberes del contrato de trabajo, sino que habría que realizar diferenciaciones respecto al contenido obligacional según las necesidades de cada relación jurídica. En definitiva, estos autores proponen una ampliación del ámbito subjetivo del Derecho del trabajo a través del establecimiento, de lo que podría llamarse, una lista de relaciones laborales especiales que no se limitarían a las relaciones laborales dependientes, sino que se incluirían las no realizadas bajo dependencia. Con ello, a modo orientativo, los autores establecen una lista de prestaciones personales de trabajo que deberían estar insertas dentro del Derecho del trabajo como, por ejemplo; las profesiones liberales, los autónomos, los consultores externos, los agentes comerciales, etc. 
protección concreto, sí fijan que, al menos, deberían tener todos derecho a la libre sindicación y a la negociación colectiva (también haciendo referencia a otros derechos individuales, pero sin matizar cuáles). En efecto, se argumenta que el Derecho del Trabajo debería aplicarse a todos aquéllos que realizan trabajo personal, esto es, que son ellos mismos los que realizan el trabajo frente a aquellos otros - los que quedarían excluidos - que, bien, tienen otros trabajadores a su cargo que realizan el trabajo por ellos, bien, es un trabajo intensivo en capital o tecnología de la cual son propietarios esos trabajadores. Esto es, el Derecho del Trabajo, de acuerdo con estos autores, debería aplicarse, con independencia de si se realiza bajo instrucciones directas o no, a todos aquéllos que prestan personalmente servicios. De esta forma, solamente quedarían excluidos aquéllos que prestan servicios a través de otras personas contratadas que los desarrollan en su nombre o aquellas que no prestan servicio personalmente, puesto que son dueños de un importante capital —en forma de tecnología o activos — siendo éste esencial para realizar el servicio.

En fin, en la doctrina comparada se puede observar un empuje hacia una redefinición del ámbito de aplicación del Derecho del Trabajo. Aunque haya diferencias entre las propuestas, todas ellas tienen un fin común: ajustar la aplicación de esta disciplina a todos aquéllos que requieren protección. Para ello se parte de la hipótesis de que en la actualidad se está excluyendo del Derecho del Trabajo a muchos sujetos que, realmente, no son empresarios. Esto es, se entiende que los límites del Derecho del trabajo deben encontrarse allí dónde se halle un genuino empresario. Por el contrario, con la actual definición de subordinación como forma de atribución de derechos laborales, el efecto generado es que existan muchos sujetos que, aunque no son genuinos empresarios, quedan excluidos del Derecho del trabajo.

En otras palabras, existen trabajadores dependientes, trabajadores independientes y empresarios. Pues bien, se defiende por estos autores que, con la configuración de la normativa actual, solamente los trabajadores dependientes quedan protegidos, no estándolo los trabajadores independientes a pesar de ser sujetos que carecen de poder de negociación suficiente como para autoprotegerse. Por ello, las propuestas pasan todas por ampliar el campo del Derecho del Trabajo, extendiéndolo a las personas que trabajan - y que no son genuinamente un empresario- aunque no se encuentren estrictamente subordinadas.

Por su parte, esta ampliación puede realizarse de dos maneras, bien mediante una modificación legislativa, bien mediante una reconfiguración interpretativa del concepto de trabajo subordinado. 


\section{Una interpretación finalista del concepto de trabajador laboral}

La idea subyacente en las propuestas presentadas hasta el momento no es otra que extender la aplicación del Derecho del Trabajo a quienes necesitan protección. Esto es, permitir que el Derecho del Trabajo satisfaga sus funciones originales. Y para ello no parece requisito indispensable un cambio normativo, sino más bien una interpretación finalista del concepto de trabajador. Como pone de relieve la situación social de muchos trabajadores actualmente clasificados como autónomos, una interpretación restrictiva de la noción de trabajador, centrada en la dependencia jurídica, excluye de su aplicación muchos trabajadores que requieren de protección. En efecto, que no exista un control directo por parte del beneficiario de una prestación de trabajo sobre el trabajador no significa que esta persona no requiera protección del Derecho del trabajo.

Por ello parece necesario realizar una interpretación teleológica del concepto de trabajador para aplicar dichas protecciones a todos aquellos que las requieran. Esto es, se pretende diferenciar un trabajador - al que se aplican las protecciones laborales - de un genuino empresario - al que no - en función de las vulnerabilidades presentes en el primero que no afectan al segundo (Davidov, 2016: 35).

La doctrina identifica tres principales razones que justifican la necesidad de la aplicación de la regulación laboral: i) fallos de mercado; ii) distribución de la riqueza; iii) desequilibrio en el poder de negociación de las partes.

En primer lugar, los fallos de mercado justifican la regulación laboral dado que el mercado laboral adolece de problemas de eficiencia que la normativa ha de solucionar. Los principales fallos que caracterizan el mercado laboral pueden resumirse de la siguiente manera (Fudge, 2006: 626): i) asimetría de la información; ii) inelasticidad en la oferta de trabajo; iii) problemas de conflicto colectivo; iv) baja inversión en el capital humano. Hyde (2006) argumenta que estos fallos de mercado afectan todas las prestaciones personales de servicio con independencia de que éstas puedan calificarse jurídicamente como subordinadas o no. Así pues, para abordar estos fallos de mercado y promover una regulación eficiente, la normativa laboral debe aplicarse a todos los trabajadores. En efecto, para combatir estos fallos es necesario que todos los trabajadores puedan organizarse y negociar colectivamente, así como tener condiciones mínimas de trabajo de carácter irrenunciable (salario mínimo, máxima jornada de trabajo, restricciones en el trabajo infantil, normativas de prevención de riesgos laborales, entre otras). En definitiva, los derechos sociales son un requisito indispensable para que funcione correctamente el mercado de trabajo (Deakin and Wilkinson 2005: 290-303), lo cual incluye a todo tipo de trabajadores sean dependientes o independientes —en tanto no sean genuinamente una empresa-.

La distribución de la riqueza y el bienestar de los trabajadores constituyen la segunda razón que justifica la legislación laboral. Prosser (2006) desarrolla la idea de 
que las condiciones laborales de los trabajadores no deben ser decididas por el mercado, sino que han de ser el resultado de un proceso deliberativo democrático. Este autor defiende la existencia de una serie de valores en la sociedad que pueden desvirtuarse si las condiciones de trabajo se dejan al libre mercado y que, por ello, se justifica la existencia de regulaciones laborales. La existencia de un salario mínimo a cambio del trabajo, la prohibición de discriminación por razones de sexo, raza, religión, el derecho a trabajar bajo medidas de seguridad que protejan a los trabajadores, una jornada máxima y los descansos, son cuestiones que el mercado por sí mismo no asegura, mientras que sí son deseados por la ciudadanía —e incluso entendidos como derechos inherentes a la condición humana-. Por ello, parece poco razonable excluir estos valores sociales y no asegurarlos normativamente simplemente por el hecho de no ser trabajador dependiente conforme a una concreta definición.

La tercera razón fundamental, y probablemente la más ampliamente aceptada, como justificación de la existencia de protección de los trabajadores es la desigualdad del poder de negociación (Davies, 1983: 18), entendida como la falta de una autonomía real cuando se trata de aceptar las condiciones de trabajo por parte de los trabajadores (Valdés Dal-Ré, 2002: 45). En efecto, igual que los trabajadores de la fábrica no podían negociar libremente sus términos contractuales ni sus condiciones laborales, dada la diferencia de poder respecto al capitalista (dueño de la fábrica), hoy en día muchos trabajadores calificados como autónomos no pueden negociar sus condiciones de trabajo. En el caso de las plataformas digitales esta cuestión parece bastante clara dado que las condiciones las fija la plataforma, siendo iguales para todos los que prestan servicios en ella, no pudiendo ser modificadas ni negociadas individualmente - contratos de adhesión-. Esto implica que los trabajadores solamente pueden aceptar o no trabajar (Murray, 2014; Hillman and Rachlinski, 2002). Lo mismo ocurre para muchos trabajadores formalmente autónomos pero que no gozan de poder para modificar los términos del contrato con su cliente por falta de poder de negociación.

Este desequilibrio en el poder de negociación parece ser la causa de todos los riesgos a los que están sometidos los trabajadores: salarios bajos, jornadas de trabajo excesivas, impredecibilidad en los horarios, transferencia de los riesgos inherentes en el negocia al trabajador, riesgos para la salud, etc. Hoy en día muchos trabajadores - sean dependientes o no- trabajan sometidos a condiciones de trabajo establecidas por una empresa sin posibilidad de ser modificadas - ni siquiera negociadas - por parte del trabajador. En vista de esta situación, el contrato de trabajo se caracteriza principalmente por normas obligatorias que no pueden ser modificadas por las partes. La legislación laboral debe imponerse a las partes, ya que el desequilibrio de poder significa que no existe una libertad contractual real en el trabajador. Las leyes laborales deben imponer límites a la aceptación de ciertas condiciones de trabajo por parte de los trabajadores siempre que medie un desequilibrio en el poder de negociación $-\mathrm{y}$ con independencia de que exista o no dependencia jurídica-. 
Al final, las tres justificaciones para la existencia del Derecho del Trabajo aquí presentadas parecen complementarias más que excluyentes. En el trabajo en plataformas, y en general en todo aquel trabajo personal que no se realiza por una empresa, existe desequilibrio de poder de negociación, fallos de mercado y necesidad de mantener ciertos valores sociales, circunstancias que justifican la aplicación del Derecho del Trabajo a esas relaciones contractuales.

Como se ha visto al principio de este artículo, la dependencia jurídica no fue originariamente - y no lo es ahora - la razón de la aparición de una gran masa de obreros que necesitaban protección. Por el contrario, fue la imposibilidad de tener en su propiedad los medios de producción relevantes. Requerir elementos productivos - activos materiales e inmateriales - de un tercero - empresario- para poder producir es lo que les hace vulnerables y los sitúa en una situación de debilidad en la negociación (desequilibrio poder de negociación). Es precisamente el hecho de que la estructura empresarial relevante no fuera de su propiedad lo que provocaba que tuvieran que cumplir las órdenes del empresario, someterse a jornadas de trabajo extenuantes, aceptar salarios por debajo del umbral de supervivencia, etc. Es decir, la jerarquía o la existencia de instrucciones por parte del empresario de obligado cumplimiento — dependencia jurídica- son un indicio de existencia de desequilibrio del poder de negociación, pero no el único.

Que actualmente exista desequilibrio de poder de negociación sin instrucciones directas no elimina la vulnerabilidad de los trabajadores, dado que esta solamente era una de las consecuencias de estar sometidos a una estructura empresarial que no le es propia. Confundir la consecuencia — dependencia jurídica - con el hecho causante de la vulnerabilidad - la falta de estructura empresarial propia y de poder de negociación - es lo que ha provocado que el derecho del trabajo deje de aplicarse a muchos sujetos que lo necesitan.

La dependencia jurídica como criterio exclusivo de aplicación del Derecho del Trabajo impide que éste cumpla con sus objetivos primigenios. La subordinación no debería ser equiparada exclusivamente con dependencia jurídica $-\mathrm{o}$ control del empresario-. Por el contrario, el ámbito de aplicación del Derecho del Trabajo debe ser interpretado en términos finalistas, esto es, como aquél que permita cumplir los objetivos de protección de quien lo necesite. Para ello se propone que el Estatuto de los Trabajadores se aplique, con independencia del nivel de control existente sobre ese trabajador, a todos aquellos que prestan servicios de forma personal en contraposición a los que prestan servicios mediante otros trabajadores o aquellos que prestan servicios mediante la propiedad de una estructura empresarial relevante. Con esta interpretación se consigue que el Estatuto de los Trabajadores se destine a todos los trabajadores que requieren protección.

Llegados a este punto se debe analizar qué significa prestar servicios de forma personal. Esta definición debe realiza en negativo, esto es, todos aquellos 
servicios que no se presten a través de una estructura productiva de la que se es titular -empresa - estarían incluidos en dicho ámbito. Aquí se revela la primera diferencia con la interpretación clásica, la cual entiende que solamente el trabajo dependiente y ajeno será laboral, quedando fuera del ámbito de aplicación el resto de tipos de trabajo.

Así pues, con la interpretación finalista se está más bien en la situación contraria: lo relevante será definir qué es una empresa, puesto que, si no se prestan servicios siendo empresario, se entenderá que se es trabajador asalariado. En este sentido, lo relevante, cuando se enfrenta a un caso de clasificación del contrato de trabajo, será la existencia de indicios de que el prestador de servicios es una genuina empresa pues lo contrario implicará que se está ante un trabajador laboral. Se entenderá que se está ante un genuino empresario cuando:

— La propiedad de activos materiales (infraestructuras, maquinaria, edificios...) o inmateriales (marca, datos, fondo de comercio, software específico, etc) pertenecen al prestador de servicios.

- La gestión real, y la toma de decisiones, de partes clave del negocio frente al mercado (precio, búsqueda y elección de clientes) es llevada por el prestador de servicios.

— La prestación de servicios es realizada por trabajadores contratados por el empresario.

En definitiva, esto es coherente con las anteriores manifestaciones realizadas sobre los nuevos indicios de laboralidad — ajenidad en la marca, ajenidad en los datos, la plataforma digital como medio de producción real, la fijación del precio del servicio, etc. - los cuales, tienen como objetivo buscar la diferencia entre un verdadero empresario de aquél que únicamente aporta trabajo ${ }^{8}$. No obstante, lo que aquí se propone es una teoría consolidada alrededor del concepto de trabajador que, a través de una interpretación finalista del concepto, permita abandonar la dependencia jurídica como criterio preponderante de la determinación de laboralidad para centrar el peso del debate en la existencia o no de una verdadera empresa: pues si el prestador de servicios no lo hace teniendo el control efectivo de una estructura empresarial relevante se entenderá que se está ante un trabajador laboral.

Además, el objetivo de esta interpretación finalista será incorporar dentro del ámbito de aplicación del Estatuto de los Trabajadores a todos los que pres-

8 En el fondo, todo ello tiene similitud a lo defendido anteriormente respecto a «los nuevos indicios de laboralidad", puesto que al final estos indicios nuevos no tienen otro objetivo que señalar la importancia que tiene en el siglo XxI unos elementos productivos distintos — ahora inmateriales principalmente - a los que tenían importancia en el siglo xx (maquinaria, fábrica). Y cómo la falta de propiedad de estos nuevos elementos productivos de relevancia es lo que realmente subordina al trabajador (ver Todoli Signes, 2018 y 2017). 
ten servicios sin ser una verdadera empresa. Ello implica que no se está aquí defendiendo una diferenciación en los niveles de protección entre un tipo de trabajadores y otros - lo que acabaría creando de nuevo trabajadores "de primera" $\mathrm{y}$ «de segunda»—. Sino una unificación —-mediante la interpretación finalista del concepto de trabajador- del régimen jurídico aplicable a todo tipo de trabajadores bajo el paraguas del Estatuto de los Trabajadores - o en caso de absoluta necesidad mediante una relación laboral especial que tenga como normativa subsidiaria el propio Estatuto de los trabajadores-.

\section{La labor del Tribunal Supremo en la reinterpretación del concepto de trabajador}

El Tribunal Supremo viene realizando, en los últimos años, una reinterpretación profunda del concepto de trabajador en nuestro país. En efecto, de forma más o menos clara, el máximo Tribunal, parece haber abandonado -o al menos reducido a la mínima expresión - la cuestión de la dependencia jurídica o el control como forma de identificar a los trabajadores.

En este sentido, en multitud de ocasiones el Tribunal Supremo ha entendido que existe contrato de trabajo aunque el trabajador tenga libertad para elegir sus propios horarios o su jornada de trabajo9. En el mismo sentido, también se ha entendido que puede existir laboralidad aunque no haya instrucciones por parte del empresario ${ }^{10}$. E incluso se entiende que la facultad del trabajador para rechazar trabajos encargados y asignados por el empresario no excluye automáticamente la laboralidad ${ }^{11}$. No obstante, es cierto que, en otras ocasiones, el hecho de que sí existan instrucciones que el trabajador deba cumplir se ha utilizado para confirmar la laboralidad ${ }^{12}$, llegando a considerar que la emisión ins-

9 STS 20 de enero de 2015 (rec. 587/2014) —caso de limpiadores de escaleras—; STS 20 de julio de 2010 (rec. 3344/2009) — limpiador de oficinas—; STS 22 de enero de 2008 (rec. 626/2007) — Transportistas con vehículo propio-; STS de 30 de abril de 2009 (1701/2008) —recogedor productos higiénicos—; STS de16 de julio de 2010 (rec. 3391/2009 y 2830/2009) de 19 de julio 2010 (rec. 1623/2009 y 2233/2009) —artistas y dobladores—; STS 3 de mayo de 2005 (rec. 2606/2004) —abogados—; STS 21 de junio de 2011 (rec. 2355/2010) —-subagentes de seguros—; STS de 14 de julio de 2016 (rec. 539/2015) —Agentes de Seguros—; STS DE 16 de noviembre 2017 (rec 2806/2015) — traductores_; STS 8 de febrero de 2018 (Rec. 3205/2015) —Montadores de ascensores-.

10 STS 20 de julio de 2010 (rec. 3344/2009) - limpiador de oficinas-; STS 22 de enero de 2008 (rec. 626/2007) - Transportistas con vehículo propio-; STS de 30 de abril de 2009 (1701/2008) — recogedor productos higiénicos—; STS de 16 de noviembre 2017 (rec 2806/2015) —Traductores_; STS 8 de febrero de 2018 (Rec. 3205/2015) —-Montadores de ascensores—.

11 STS de 16 de noviembre 2017 (rec 2806/2015) -Traductores_; STS de 16 de julio de 2010 (rec. 3391/2009 y 2830/2009) de 19 de julio 2010 (rec. 1623/2009 y 2233/2009) —artistas y dobladores-.

12 SSTS de 10 de julio del 2000 (rec 4121/1999); 15 de octubre de 2001 (rec. 2238/2000) y 7 de octubre de 2009 rec. 4169/2008) 
trucciones por parte del empresario "para una correcta ejecución del servicio» es una intromisión ilegítima en el ámbito de actuación de un empresario que se debiera considerar independiente, calificándolo por ello de laboral (STS de 24 de junio de 2015 rec. 1433/2014). Por tanto, los clásicos indicios de la dependencia como son la fijación de horarios y de instrucciones parecen servir para confirmar la laboralidad pero no para excluirla cuando se dan otros indicios. En efecto, desde hace tiempo, la doctrina defiende una flexibilización del concepto «dependiente» para entender cumplido este requisito por el hecho de estar inserto en la organización empresarial (Rodríguez-Piñero; 1966).

Respecto a la ajenidad, es de sobra conocido como el Tribunal Supremo mantiene que la propiedad de herramientas de trabajo o de medios de producción secundarios (o no relevantes) no excluyen la calificación de trabajador laboral $^{13}$. De hecho, incluso asumir parte del riesgo ${ }^{14}$ no es impedimento para la laboralidad. Tampoco que quede acreditado que no existe exclusividad - dependencia económica-,y que quede atestiguado que realizaba trabajos para otras empresas, ha evitado que calificara el contrato como de trabajo ${ }^{15}$.

Así pues, el Tribunal Supremo está abandonando progresivamente, bajo mi punto de vista, una interpretación rígida de la dependencia y la ajenidad por la cual solamente aquéllos que cumplan cumulativamente los dos requisitos - amén de la voluntariedad, la retribución y el trabajo personal— serán trabajadores laborales, adoptando una interpretación amplia del trabajo subordinado como toda aquella prestación personal de servicios ${ }^{16}$. A su vez, parece que el concepto de prestación personal de servicios viene definido en contraposición a la prestación de servicios con una organización empresarial propia.

Por supuesto, siguen existiendo dudas interpretativas, puesto que no será siempre fácil definir qué se entiende por «organización empresarial propia». No obstante, también aquí el Tribunal Supremo, en alguna sentencia reciente, deja elementos suficientes apuntados.

13 STS 20 de enero de 2015 (rec. 587/2014) —caso de limpiadores de escaleras_; STS 20 de julio de 2010 (rec. 3344/2009) —compra propia de elementos de limpieza—; STS 8 de febrero de 2018 (Rec. 3205/2015), STS 22 de enero de 2008 (rec. 626/2007) — propiedad de furgoneta-; 7 de octubre de 2009 rec. 4169/2008) —alquiler de oficina y pago secretaria-.

14 STS 9 de marzo de 2010 rec. 1443/2009). En este caso, se descontaban los gastos de mantenimiento de la clínica dental.

15 STS 22 de enero de 2008 (rec. 626/2007) — Transportistas con vehículo propio_; STS de 30 de abril de 2009 (1701/2008) — recogedor productos higiénicos—; STS 3 de mayo de 2005 (rec. 2606/2004) —abogado-.

16 Obviamente esta evolución no está exenta de contradicciones y pasos atrás, por ejemplo, la STS 7 de octubre de 2009 (rec. ud. 4169/2008) establece que la nota distintiva decisiva debe ser la dependencia y no la ajenidad, decantándose con ello por el criterio de la dependencia jurídica. Un criterio que en otras ocasiones descarta al afirmar que el hecho de que no existan instrucciones ni horarios y que el trabajador pueda libremente rechazar trabajos no impide la calificación de laboralidad (STS de 16 de noviembre 2017 (rec 2806/2015) —-traductores). 
Se está haciendo referencia a la STS de 16 de noviembre 2017 (rec 2806/2015) del caso Ofilingua - en materia de traductores-. En efecto, en esta sentencia se enjuicia un caso en el que los trabajadores - formalmente autónomos- gozan de libertad de horarios y de jornada pudiendo rechazar — sin consecuencias negativas - los encargos de la empresa, no existe formación por parte de la empresa, no se asigna ni se paga vacaciones (y ni siquiera exige la empresa que se le comunicara tales circunstancias por parte del trabajador), no hay instrucciones sobre cómo realizar el trabajo, no hay exclusividad y los trabajadores realmente realizan otras tareas - de traducción o interpretación - para otros empresarios o clientes, tampoco la empresa aporta material alguno para desarrollar su prestación de servicios, teniendo el trabajador que hacerlo si lo necesita (material de oficina, papel, bolígrafos, ordenador, teléfono, diccionario, internet, glosarios, vehículo, etc.). Pues bien, a pesar de esa falta de dependencia jurídica — mostrada claramente desde el momento en que no existen instrucciones y, además, el trabajador puede rechazar los encargosel Tribunal Supremo entiende que existe laboralidad.

Para alcanzar esta conclusión, el Tribunal se sirve de seis criterios, que ahora se pasan a exponer. No obstante, como luego se verá, pueden resumirse en dos.

1) En primer lugar, se entiende que existe laboralidad, dada «la adopción por parte del empresario - y no del trabajador- de las decisiones concernientes a las relaciones de mercado o con el público».

2) En segundo lugar, la empresa es la que fija los precios o tarifas del servicio. Es decir, el precio del trabajo no es negociado con el trabajador presuntamente autónomo, sino que era un «o lo tomas o lo dejas» impuesto por el empresario.

3) En tercer lugar, la empresa es la que seleccionaba los clientes finales o personas a las que atender.

4) En cuarto lugar, el Tribunal Supremo entiende que existe laboralidad, puesto que «el cálculo de la retribución (se realiza) con arreglo a un criterio que guarde una cierta proporción con la actividad prestada». Esto es, la retribución está conectada con las horas de trabajo y no con otros criterios empresariales.

5) En retribución percibida por el trabajador se nota la ausencia «del lucro especial que caracterizan a la actividad del empresario o al ejercicio libre de las profesiones».

6) Por último, el Tribunal Supremo concluye que existe laboralidad puesto que el trabajador presta servicios con «inexistencia de estructura empresarial».

Como se puede apreciar, son seis criterios bien argumentados pero que no apuntan a una dependencia jurídica clásica, sino que más bien se dirigen a demostrar que estos trabajadores que prestaban servicios no eran una genuina empresa. 
De esta forma, parece que efectivamente nuestro Tribunal Supremo acoge esa doctrina por la cual el ámbito del Derecho del trabajo no ha de limitarse al trabajo dependiente en sentido estricto, sino a toda prestación personal de servicios que no se haga como un genuino empresario. Es decir, partiendo de la premisa de que existe una amplia gama de tipos de prestación de servicios — trabajo dependiente, el trabajo independiente y los empresarios-,el Tribunal Supremo, con una interpretación finalista, acoge dentro del ámbito del contrato de trabajo a los dos primeros supuestos, excluyendo solamente al tercero.

De hecho, en mi opinión, aunque es cierto que el Tribunal Supremo recoge seis indicios para defender la laboralidad, estos argumentos van todos dirigidos a un objetivo: demostrar que estos trabajadores no eran una empresa real. Así, que los trabajadores no tomaran decisiones respecto al mercado y con el público, que no seleccionaran la clientela o el personal a atender, que la fijación de precios o tarifas viniera impuesta, que la retribución dependiera de las horas de trabajo, son indicios, no tanto de que el trabajo es subordinado, sino de que; i) no pueden negociar sus propias condiciones de trabajo; ii) no son una empresa, puesto que no se encargan de una de las partes más importantes de todo negocio - márquetin, conseguir clientes, mantenerlos, decidir precios, etc.-

A mayor abundamiento, la «inexistencia de estructura empresarial» es recalcada por la propia sentencia de nuevo para concluir que eran trabajadores laborales. La falta de elementos que determinen que se está ante una verdadera empresa - como la inexistencia de áreas importantes en todo negocio, pero también la falta de una estructura de activos (materiales o inmateriales) importante- parece lo determinante. En efecto, en este caso, la necesidad de disponer de un ordenador para realizar el trabajo no es suficiente para entender que la prestación requiere alta inversión en activos realizada por quien presta el servicio ${ }^{17}$. Así pues, si la prestación de servicios requiere activos de capital económicamente relevantes, éstos pertenecen al prestador de servicios y, además, cuenta con las áreas necesarias para explotar el negocio, se le podría considerar un empresario.

Respecto al indicio de ausencia del lucro especial que caracteriza a la actividad del empresario o el ejercicio libre de las profesiones puede estar remitiendo a dos cuestiones. La primera podría ser respecto a que la percepción recibida en forma de salario es baja, sin embargo, no parece que tener un salario alto excluya la laboralidad — ni siquiera en casos complejos_- dado que en el conocido caso del tertuliano radiofónico la retribución era muy superior a la media española y

17 Un nivel relevante de activos que sí podrían excluir la laboralidad podría ser la inversión propia en un local, maquinaria o elementos de capital necesarios para realizar la actividad (ej. un restaurante que requiere local, reformas, cocina, etc...). La propiedad de una marca relevante, un software específico valorado por el mercado, patentes, propiedad intelectual u otros activos inmateriales relevantes o incluso suficiente información o datos que asegure una diferenciación en el mercado. 
se entendió que se trataba de una prestación laboral de servicios (STS 19 febrero 2014, rec. 3205/2012).

La otra posibilidad es que esté haciendo referencia a la plusvalía. Esto es, al concepto que en economía es definido como el rendimiento obtenido por el empresario derivado del aprovechamiento de la mano de obra ajena. En este sentido, a lo que podría estar aludiendo el Tribunal Supremo es que estos trabajadores percibían una retribución por su prestación personal de servicios y no por gestionar una empresa en la cual otros, contratados por un genuino empresario, prestaban el servicio.

En mi opinión, esta segunda tiene mayor lógica puesto que, si no existen trabajadores a su cargo, significa que esa persona presta todo el servicio ella misma, difícilmente constituyendo eso una verdadera empresa. Además, otros Tribunales extranjeros vienen afirmando, en este sentido, que un autónomo no solamente debe tener una retribución proveniente de algo distinto a su propia mano de obra, sino que además debe poder tener unas verdaderas perspectivas de crecimiento del negocio más allá de percibir más dinero porque trabaja más horas ${ }^{18}$.

En definitiva, y siempre con todas las cautelas, el Tribunal Supremo parece realizar una interpretación finalista del contrato de trabajo para aplicarse a todo aquel que preste servicios de manera personal, sea subordinado o no. Pocas dudas caben de que si el trabajador solamente aporta mano de obra no puede ser un empresario y, por ello, se le debe aplicar las protecciones laborales. Sin embargo, sigue existiendo la duda de qué es un genuino empresario.

Para ello, el Tribunal Supremo parece fijarse en dos características: primero, que tenga estructura empresarial haciendo referencia, de un lado, a elementos materiales (maquinaria, edificios...) o inmateriales (marca, datos, fondo de comercio, software específico, etc.) de relevancia que pertenecen al que presta el servicio $^{19} \mathrm{y}$, de otro lado, que tenga una organización suficiente que contemple no solamente la ejecución de la prestación sino otros elementos inherentes a la gestión de un negocio (política de márquetin, gestión del negocio, toma de deci-

18 Employment Tribunal UK case Mr Y Aslam, Mr J Farrar and Others - V- Uber Case Numbers: 2202551/2015 Comentada en https://adriantodoli.com/2016/11/02/comentario-a-lasentencia-que-declara-la-laboralidad-de-los-conductores-de-uber-en-uk/

19 En el fondo, todo ello tiene similitud a lo defendido anteriormente respecto a «los nuevos indicios de laboralidad" puesto que al final estos indicios nuevos no tienen otro objetivo que seńalar la importancia que tiene el S. xxi unos elementos productivos distintos — ahora inmateriales principalmente- a los que tenían importancia en el S. xx (maquinaria, fábrica), ver Todoli Signes (2018). También las sentencias que declaran la laboralidad del trabajo en plataformas digitales han llegado a esta conclusión al entender que los «riders» no son verdaderos empresarios. Esto es así en la Sentencia del Juzgado de los Social el juzgado n. ${ }^{\circ} 6$ de Valencia n. ${ }^{\circ}$ 244/2018 de 1 de junio y Sentencia del Juzgado de lo Social n. 33 de Madrid de 11 de febrero, la Sentencia del Juzgado de los Social n. ${ }^{\circ} 1$ de Gijon n. ${ }^{\circ}$ 61/2019, Juzgado de lo Social n. ${ }^{\circ} 1$ de Madrid en Sentencia n. ${ }^{\circ} 130$ de 2019 de 4 de abril. En la doctrina se puede consultar, Todolí Signes, (2018b), Sánchez Pérez, (2019) 
siones relevantes, etc... $)^{20}$. Segundo, se entiende que será empresario aquél que tenga trabajadores a su cargo que sean los que realmente realicen el trabajo por el cual el genuino empresario recibe sus beneficios.

En fin, con esta interpretación finalista se consigue que el Derecho del Trabajo cumpla sus fines en materia de fallos de mercado, cumplir con los valores sociales y proteger a la parte débil del contrato que carece de poder de negociación para tener una verdadera autonomía de la voluntad cuando acepta las condiciones de trabajo.

\section{Reflexión final}

La tecnología, en los próximos años, va a modificar completamente la forma en que muchos tipos de empresas interactúan en el mercado, volviendo cada vez menos necesaria la figura del trabajador jurídicamente dependiente. En ciertos sectores — principalmente de servicios - las empresas no van a necesitar dirigir y supervisar el trabajo realizado. Por el contrario, las empresas, a través de la tecnología, van a confiar en las evaluaciones realizadas por sus clientes de los resultados del trabajo. Con esas mismas evaluaciones se seleccionarán a los futuros trabajadores (control ex ante y control ex post). Ni siquiera las empresas tendrán la necesidad de formar a sus trabajadores pues éstos, si desean trabajar, habrán de estar formados y listos para el trabajo. El trabajo moderno, a través de redes virtuales, y con trabajadores de mayor cualificación, se configura con una menor dependencia y una mayor libertad para el trabajador a la hora de realizar su trabajo. Esta situación puede conllevar que la actividad no siempre encaje perfectamente con la definición de contrato de trabajo existente. No obstante, no debería deducirse que este nuevo tipo de trabajadores no necesiten protección.

El trabajador subordinado es el centro de la protección laboral, por lo que de no cambiar nada, el Derecho del trabajo puede quedarse sin sujeto jurídico que proteger. Como dijo Romagnioli (1999: 10) «en los años 60 y 70 el Derecho del trabajo italiano entró en la fábrica (...), pero las fábricas se han vaciado. De improvisto y bastante deprisa». Ahora mismo, nos enfrentamos al mismo fenómeno, pero llevado a su máximo exponente.

En el futuro, la tecnología, y el nivel de formación de las personas, reducirá el uso de las instrucciones directas como forma de subordinar el trabajador. Sin

20 El Tribunal Supremo para distinguir entre una falsa cooperativa y una verdadera cooperativa, a efectos de conocer si los trabajadores cooperativistas eran verdaderos cooperativistas o falsos autónomos también recurre, una vez más, a la cuestión del trato con los clientes y la gestión del negocio. Entendiendo que si la cooperativa no realiza funciones de este tipo se estará ante una falsa cooperativa —, ver STS de 18 de mayo de 2018 (rec.3513/2016). 
embargo, que los trabajadores carezcan de supervisión o control directo no significa que no requieran protección de las normas laborales - derecho vacaciones, salario mínimo, protección de riesgos laborales, etc.- - Por ello, parece necesaria una interpretación teleológica del ámbito de aplicación del Derecho del trabajo que haga posible que éste cumpla las funciones para las que nació. Difícilmente se puede sostener que, actualmente, muchos de los autónomos existentes realmente puedan negociar sus condiciones de trabajo. Más bien al contrario, existe un desequilibrio que requiere que éstos acepten o no trabajen.

Por ello, se propone que el Derecho del Trabajo se aplique a quienes prestan servicios de forma personal - en contraposición a aquéllos que lo hacen siendo propietarios de una estructura empresarial-. En este trabajo, se sostiene que el Tribunal Supremo ha iniciado una reinterpretación del concepto de trabajador, aportando sentencias que lo muestran. En efecto, partiendo de la premisa de que en el espectro de la prestación de servicios cabe el trabajo dependiente, el trabajo independiente y los empresarios, el Tribunal Supremo, sirviéndose de una interpretación finalista, parece acoger en los últimos años dentro del ámbito del contrato de trabajo a los dos primeros supuestos, excluyendo solamente al tercero.

Actualmente, muchos autónomos no pueden calificarse de verdaderos empresarios en el sentido de poder negociar en igualdad de condiciones y usar su iniciativa para maximizar sus beneficios. En cambio, son mano de obra fungible dentro de una larga lista de demandantes de trabajo. Si no encajan en la interpretación, doctrinal y judicial, clásica del concepto de trabajador no es porque no se enfrenten a la misma realidad social, sino porque dicha construcción doctrinal y judicial nació para ser aplicada a una realidad distinta a la actual. Sin embargo, nada impide mediante una interpretación finalista, se consiga que el concepto de trabajador protegido abarque a todos aquellos que requieren de ella.

Más aún en el caso de algunos concretos derechos laborales recogidos en la propia Constitución española. En efecto, la libertad sindical, la negociación colectiva y el derecho de huelga viene reconocidos a todos los trabajadores — sin que la letra de la norma distinga entre subordinados o independientes-. Que de estos derechos se excluya a los trabajadores independientes es una interpretación restrictiva del concepto trabajador no justificada ni por la realidad social en la que vivimos — necesidad de los autónomos de agruparse para mejorar su poder de negociación - ni por las funciones atribuidas a estos derechos — valores democráticos en el trabajo y resolución de fallos de mercado- y mucho menos dado el carácter de derecho fundamental que le otorga la propia Carta Magna.

En fin, con una interpretación finalista del concepto de trabajador, esta es, aquella que permite proteger a la parte contractual débil de una prestación de servicios, mantener los valores sociales y reparar los fallos de mercado existentes en el mundo laboral, equivalente a incluir dentro de su ámbito toda prestación de servicios no realizada por una auténtica empresa, se conseguiría dar seguridad 
jurídica actualizando el concepto de trabajador y adaptándolo a la realidad social y productiva de nuestra época.

El caso más palmario de esta necesidad son los trabajadores de plataformas. Por mucha libertad de horarios, y falta de instrucciones que formalmente gocen, se encuentran sometidos a los deseos de la plataforma. La razón fundamental de ello es no son verdaderos empresarios con poder de negociación propio. Sin embargo, en el presente trabajo no se busca dar una solución aplicable exclusivamente a los trabajadores en plataformas, sino a todos aquellos prestadores personales de servicios - sean dependientes o independientes - que carecen de poder de negociación frente a los beneficiarios de sus servicios. Es para proteger a éstos para lo que nació el Derecho del trabajo.

\section{Bibliografía}

BBVA (2018), La participación del trabajo en la renta nacional, https://www.bbva.com/es/ la-participacion-del-trabajo-en-la-renta-nacional/ (consultado 10 de abril de 2019)

Calvo Rodríguez E., (2017). La responsabilidad empresarial en la subcontratación laboral: análisis del articulo 42 del Estatuto de los Trabajadores, Tesis doctoral, Valencia.

Countouris y De Stefano (2019), "New forms of employment», Syndicat Europeaen Trade Union.

Davidov, (2002), The three axes of employment relationships: A characterization of workers in need of protection, U.TL.J., 52.

Davidov, (2016), A Purposive Approach to Labour Law, Oxford, 35-45.

Davies, Freedland, Kahn-Freund, (1983), Labour and the Law, Stevens, 18.

De Stefano V., "The rise of the «just-in-time workforce», ILO, available https://www. ilo.org/wcmsp5/groups/public/---ed_protect/---protrav/---travail/documents/publication/wcms_443267.pdf

DeAKIN y Wilkinson, (2005), The Law of the Labour Market: Industrialization, Employment, and Legal Evolution, Oxford, pp 290-303.

Dockés (ed.) (2017), Proposition de Code du Travail, Dallorz, 2017.

EwING, Hendy y Jones (2016), A manifesto for Labour Law: towards a comprehensive revision of worker's right, IER, $\mathrm{p} 35$.

Ewing, Hendy y Jones (2018), Rolling Out the Manifesto for Labour Law, IER.

Freedland M y Kountouris N., L., (2011), The legal construction of personal work relations, Oxford.

FUdGE, (2006), Fragmenting work and fragmenting organizations: The contract of employment and the scope of labour regulation, OHLJ, 44, 4.

Goos, M., et al, (2019) «The impact of Digital Transformation on EU Labour Markets», European Commission.

Hugh Collins, (2000), "Justification and techniques of legal regulation of the employment relations», in Hugh Collins, Paul Davies \& Roger Rideout (eds.), Legal Regulation of the Employment Relation, Kluwer International, 7-11 (2000) and

Hyde, (2006) "What is Labour Law», in Davidov \& Langille (eds.), Boundaries and Frontiers of Labour Law, 37-38. 
JPMorgan Chase (2018), Uber drivers are earning 53\% less than they did in 2013 https://bigthink.com/technology-innovation/uber-drivers-earning-less

Lyon-Caen G., Le droit du trevail non salarié, Sirey, Paris, 1990.

Pérez De Los Coвos, F., (1993) «El trabajo subordinado como tipo contractual», Documentación Laboral, N. ${ }^{3} 39,1993$.

Perez Garcia F., et al., (2019), "Cambios tecnológicos, trabajo y actividad empresarial: el impacto socioeconómico de la economía digital», XXI Premio de Investigación del CES (en prensa).

Pesole, A., Urzí Brancati, M.C, Fernández-Macías, E., Biagi, F., González VÁzquez, I. (2018), Platform Workers in Europe Evidence from the COLLEEM Survey, Publications Office of the European Union.

Prosser (2006), "Regulation and Social Society», Journal of Law and Society, 33, 3, 364-387.

Rodríguez-Piñero, M., «La dependencia y la extensión del ámbito del Derecho del Trabajo» RPS, 71, 1966.

Romagnoli, U., "Globalización y Derecho del Trabajo», Revista de Derecho Social, 5, 1999.

SÁnchez PÉrez, J. «Economía colaborativa y crisis del derecho del trabajo», Estudios Financieros. Revista de Trabajo y de Seguridad Social (RTSS. CEF), núm. 430, Enero 2019, pag. 75 a 100.

Supiot A., et al., (2001), Beyond Employment —Changes in work and the Future of Labour Law in Europe, Oxford.

Tegmark M., Life 3.0: Being Human in the Age of Artificial Intelligence, KNOPF, 2017, p 121.

Todoli Signes, A., (2018), «Prólogo», en Todoli Signes A., Hernadez Bejarano (Dir.), El trabajo en plataformas digitales, Aranzadi.

Todolí Signes, A., (2018b), "Comentario a la sentencia que califica como trabajador a un rider de Deliveroo y sus efectos en la gig economy», Revista General de Derecho del Trabajo y de la Seguridad Social, N.o. 51, págs. 443-447

Todoli Signes, A., (2017), El trabajo en la era de la economía colaborativa, Tirant lo Blanch.

VALDÉs DAL-RE, (2002), «Descentralización productiva y desorganización del derecho del trabajo", Sistema: Revista de Ciencias Sociales 168-169, 61. 